\title{
Left Ventricular Hypertrophy and Antiarrhythmic Drugs In Atrial Fibrillation: Impact On Mortality
}

\author{
Roy Chung, MD, Penny L. Houghtaling, MS, Michael Tchou, MD, Mark J. Niebauer, MD, \\ PhD, Bruce D. Lindsay, MD, Patrick J. Tchou, MD, and Mina K. Chung, MD \\ The Department of Cardiovascular Medicine, Heart and Vascular Institute, Cleveland Clinic, \\ Cleveland, $\mathrm{OH}$
}

\section{Abstract}

Background-Despite sparse clinical data, current atrial fibrillation (AF) guidelines favor amiodarone as a drug of choice for patients with left ventricular hypertrophy (LVH).

Objective-This study tested the hypothesis that patients with persistent AF and LVH on nonamiodarone antiarrhythmics have higher mortality compared to patients on amiodarone.

Methods-In an observational cohort analysis of patients who underwent cardioversion for AF, patients with LVH, defined as left ventricular wall thickness $\geq 1.4 \mathrm{~cm}$, by echocardiogram prior to their first cardioversion were included; clinical data, including antiarrhythmic drugs and ejection fraction (LVEF) were collected. Mortality, determined via the Social Security Death Index, was analyzed using Kaplan-Meier and Cox proportional hazards models to determine whether antiarrhythmic drugs were associated with higher mortality.

Results-In 3926 patients, echocardiographic wall thickness was available in 1399 (age $66.8 \pm 11.8$ years, $67 \%$ male, LVEF $46 \pm 15 \%$, septum $1.3 \pm 0.4$, posterior wall $1.2 \pm 0.2 \mathrm{~cm}$ ), and $537(38 \%)$ had $\mathrm{LVH} \geq 1.4 \mathrm{~cm}$. Among 537 patients with $\mathrm{LVH}$, mean age was $67.5 \pm 11.7$ years, $76.4 \%$ were males, and mean LVEF was $48.3 \pm 13.3 \%$. Amiodarone was associated with lower survival (log rank $\mathrm{p}=0.001)$, including after adjusting for age, $\mathrm{LVEF}$ and CAD $(\mathrm{p}=0.023)$. In propensity-score matched cohorts with LVH treated with no drugs, non-amiodarone antiarrhythmic drugs or amiodarone ( $\mathrm{N}=65$ each group), there was early lower survival in patients on amiodarone $(\mathrm{p}=0.05)$.

Conclusions-Patients with persistent AF and LVH on non-amiodarone antiarrhythmic drugs do not have higher mortality compared to patients on amiodarone. Importantly, these findings do not support amiodarone as a superior choice in patients with LVH.

\section{Keywords}

Left Ventricular Hypertrophy; Atrial Fibrillation; Antiarrhythmic drugs; Mortality

Address correspondence to: Mina K. Chung, M.D. Department of Cardiovascular Medicine Heart and Vascular Institute Cleveland Clinic 9500 Euclid Avenue, J2-2 Cleveland, OH 44195 chungm@ @ccf.org Phone: 216-444-2290 FAX: 216-636-6951.

CONFLICT OF INTEREST DISCLOSURES

The authors report no conflicts of interest. 


\section{INTRODUCTION}

Although hypertension and left ventricular hypertrophy $(\mathrm{LVH})$ are predictors of atrial fibrillation (AF) occurrence, ${ }^{1-4}$ The safety of antiarrhythmic drugs and therapeutic management of AF in patients with LVH have not been well established. ${ }^{5}$ Current American College of Cardiology (ACC)/American Heart Association (AHA)/European Society of Cardiology (ESC) 2006 guidelines for the management of AF recommend amiodarone as the first line and only drug listed in an algorithm of drug treatment for patients with $\mathrm{LVH}$ and AF. The ACC/AHA/Heart Rhythm Society (HRS) 2011 focused update to the 2006 guidelines continues to only list amiodarone for patients with LVH and AF, and the ESC 2010 guidelines for management of AF suggest that dronedarone may be an alternative, although there is a scarcity of clinical data to support these recommendations. ${ }^{6-8}$ The assumption that amiodarone compared to nonamiodarone antiarrhythmic drugs would produce a more favorable prognosis is based on a theoretical presumption that amiodarone has less risk for proarrhythmia in LVH populations with AF. However, following a thorough Pub Med review, we were unable to find any clinical studies in humans to substantiate a literature basis for this recommendation.

Therefore, the objective of this study was to assess the impact of amiodarone versus nonamiodarone antiarrhythmic drugs (AADs) on survival in patients with AF and LVH in a cohort of patients with echocardiographic evidence of $\mathrm{LVH}$ and persistent $\mathrm{AF}$ undergoing cardioversion.

\section{METHODS}

This study was a retrospective observational cohort analysis of consecutive patients who underwent cardioversion for AF and who had echocardiograms with left ventricular wall thickness data.

\section{Patient Population}

At the Cleveland Clinic all patients undergoing procedures in the Cardiac Electrophysiology Laboratory are entered prospectively into a database. From this database a research database of all patients who underwent cardioversion for AF between August, 1996, and November, 2003, was formed and demographic, clinical, echocardiographic, laboratory, and long-term survival data collected. Consecutive patients were included at their first cardioversion in the database if they had an echocardiogram prior to their cardioversion. The study was approved by the Cleveland Clinic Institutional Review Board for medical records review and performed in accordance with institutional guidelines.

\section{Definitions}

In the AF management guidelines, the definition of LVH was revised from a left ventricular wall thickness wall of $\geq 1.4 \mathrm{~cm}$ in 2001 to substantial LVH with no clear defining wall thickness in 2006. ${ }^{9}$ Based upon the 2001 definition, $\mathrm{LVH}$ was defined as a septal or posterior wall thickness of $\geq 1.4 \mathrm{cmfor}$ the primary analyses for this study. 


\section{Antiarrhythmic Drug Use Categorization}

The cohort was categorized based on AAD use, using the Vaughn-Williams classification. Class IA drugs included procainamide, quinidine, and disopyramide. Class IC drugs included flecainide, propafenone, and moricizine. Class III drugs included sotalol, dofetilide, and amiodarone. For the primary analyses, patients were stratified into 3 exposure groups, based on AAD use at the time of first cardioversion in the database: No Vaughn-Williams Class I or III antiarrhythmic drugs (No AAD), non-amiodarone Class I or III antiarrhythmic drugs (Non-Amiodarone AAD), and amiodarone (Amiodarone). Since dronedarone was unavailable during the study period, no dronedarone-treated patients were included in our analyses.

\section{Data Collection and Follow up}

Data collected included medications at the time of cardioversion, cardiovascular diagnoses, echocardiographic left ventricular wall thickness (interventricular septum and posterior wall) and left ventricular ejection fraction (LVEF). Mortality was determined from query of the Social Security Death Index (SSDI). For surviving patients, a follow up time was calculated based on the latest query date of February 8, 2010, censoring patient follow-up on October 8,2009 , to account for lag time from death to reporting in the SSDI. Patients without Social Security numbers were excluded from mortality analyses.

\section{Statistical Analysis}

Continuous variables are expressed as mean \pm standard deviation, unless otherwise specified. Continuous variables were analyzed using two-tailed Student's $t$-tests and categorical variables using chi-square or Fisher's exact tests. Survival analyses were performed timed from the first cardioversion in the database. Variables with univariate statistical significance $\mathrm{p}<0.10$ or clinical relevance were tested in a forward stepwise multivariate fashion using a Cox proportional hazards regression method in order to find predictive models for mortality. Variables analyzed included: age, sex, LVEF, coronary artery disease (CAD), valvular heart disease, non-ischemic cardiomyopathy, and smoking history. Results are reported with hazard ratio (HR), 95\% confidence interval (CI) and p value for each significant variable. Primary analyses were performed comparing the three AAD groups (No AAD, Non-Amiodarone AAD, and Amiodarone), as well as in AAD users only (Non-Amiodarone AAD and Amiodarone). Subanalyses were also performed by individual AADs and by class I and non-amiodarone class III AADs, compared to amiodarone. Overall and stratified nonparametric survival estimates were obtained by the method of Kaplan and Meier. SPSS version 11.5 for Windows XP and SAS version 9.2 were used for statistical analyses. $P$ values $<0.05$ were considered statistically significant.

To adjust for inherent differences between those patients receiving amiodarone or not, and to more accurately compare groups, propensity matching was employed. Multivariable logistic regression analyses were performed to determine factors associated with use of amiodarone compared to other treatments. The parsimonious predictive model was augmented with other available factors to form a propensity model. Solving the equation for each patient yielded a propensity score (the probability of being on amiodarone). This propensity (balancing) score was then used to find well matched controls with the other 
group, using greedy matching closest matches within a maximum distance of 0.2. Following matching, patients were sorted based on propensity scores and divided into quintiles. Characteristics were evaluated to ensure balanced factors in the groups within similar propensity score ranges. The resulting matched patients were used in subsequent analyses.

\section{RESULTS \\ Baseline Characteristics}

Among 3926 patients who underwent their first cardioversion for atrial fibrillation in our institution between March 1996 and November 2003, there were 1399 patients who had echocardiographic data available prior to cardioversion. In the total cohort, mean age was $66.8 \pm 11.8$ years, $975(70 \%)$ were male, 537 (38\%) had LVH $\geq 1.4 \mathrm{~cm}, 862(62 \%)$ had no LVH $(<1.4 \mathrm{~cm}), 21.6 \%$ were on amiodarone, and the mean LVEF was $46.1 \pm 14.6 \%$. Mean septal wall thickness was $1.28 \pm 0.25 \mathrm{~cm}$ and mean posterior wall thickness $1.19 \pm 0.35 \mathrm{~cm}$ for the whole cohort. In the LVH cohort, mean septal thickness was $1.57 \pm 0.36 \mathrm{~cm}$ and mean posterior wall thickness $1.39 \pm 0.24 \mathrm{~cm}$.

Patients with LVH were more likely to be male, have higher LVEF and larger left atrial size, but smaller LV dimensions. Patients on amiodarone with or without LVH were more likely to have structural heart disease, including CAD, non-ischemic cardiomyopathy, lower LVEF, and larger left atrial and left ventricular dimensions, when compared to patients on non-amiodarone AADs (Tables 1 and 2). Cardioversion was successful in patients without LVH, 89\% (768/862) of patients without LVH and in 90\% (485/537) of patients with LVH.

\section{Survival analyses}

During the follow up period, 274/537 (51\%) patients with LVH died over a mean follow-up time of $7.1 \pm 3.5$ (median 7.5) years. Kaplan Meier survival analysis of the patients with LVH stratified by AAD treatment (Figure 1A) suggested significant survival differences between the 3 different exposure groups ( $\log$ rank $\mathrm{p}=0.001$ ). Estimated unadjusted 1,5 , and 10 year survival for the no AAD group was $93 \%, 69 \%$, and $46 \%$; in the non-amiodarone AAD group $96 \%, 84 \%$, and $60 \%$; and in the amiodarone group $90 \%, 58 \%$, and $35 \%$, respectively. As amiodarone therapy was associated with a higher risk group of patients, as evidenced by the higher prevalence of $\mathrm{CAD}$, non-ischemic cardiomyopathy and lower LVEF, we attempted to adjust for these differences using Cox proportional hazards regression modeling. After adjusting for factors known to be associated with poorer outcomes, such as age, LVEF, CAD and smoking history, there were no significant differences between the three groups ( $\mathrm{p}=0.63$ ), but significantly worse survival in the amiodarone cohort when compared to the nonamiodarone AAD group remained $(\mathrm{p}=0.023)$. Among patients with LVH taking AADs, significant independent predictors of mortality in a multivariate analysis included older age, lower LVEF, smoking history, and amiodarone use (Table 3).

The non-amiodarone AAD groups were sub-categorized to Class 1A, Class $1 \mathrm{C}$ and nonamiodarone Class 3 AAD groups. In a Kaplan Meier survival analysis (Figure 1B), when compared to amiodarone, Class 1A and non-amiodarone Class 3 AADs showed a trend 
toward better survival, but with no statistical significance on Cox adjusted analysis ( $\mathrm{p}=0.25$, $\mathrm{p}=0.11$ respectively). However, Class $1 \mathrm{C}$ AADs were associated with better survival when compared to amiodarone (Cox adjusted $\mathrm{p}=0.032$ ).

\section{Analysis of survival in patients without LVH}

To determine if survival differences between amiodarone and non-amiodarone AAD groups were specific for patients with $\mathrm{LVH}$, we analyzed the survival of patients without $\mathrm{LVH}$. There were 420 patients without LVH who were on AADs, with 187 on amiodarone and 233 on non-amiodarone AADs. Over a mean follow up of 7.4 \pm 3.1 (median 7.5) years, $53 \%$ of this population died, 103 (46\%) on non-amiodarone, 120 (64\%) on amiodarone and 230 (52\%) on no AADs. The amiodarone group had more males, smoking history, CAD, nonischemic cardiomyopathy, and lower EF than the non-amiodarone group. Unadjusted survival estimates at 1, 5, and 10 years for the no AAD group were $94 \%, 78 \%, 56 \%$; in the non-amiodarone group 97\%, 82\%, 63\%; and in the amiodarone group $84 \%, 61 \%, 40 \%$, respectively. There were significant differences in survival among the unmatched groups ( $\mathrm{p}<0.0001)$, similar to those noted in the LVH cohorts.

\section{Propensity Score Matched Group Analyses}

Patients on amiodarone tend to have lower LVEF, higher prevalence of CAD and other comorbidities. In order to robustly test the hazards of amiodarone exposure in patients with $\mathrm{AF}$ and $\mathrm{LVH}$, we performed analyses in propensity score matched groups. Propensity matching was performed based on the following variables: sex, age, body mass index, race, history of CAD, valvular heart disease, non-ischemic cardiomyopathy, hypertrophic cardiomyopathy, tobacco exposure, alcohol use, LVEF, septal wall thickness and posterior wall thickness. Using propensity score matching, matches were found for 65 patients on no AAD, 65 patients on amiodarone and 65 patients on non-amiodarone AADs. Table 4 shows no significant differences in the matched characteristics between groups. A covariance plot (Figure 2) shows standardized differences in patient characteristics between the nonamiodarone and the amiodarone groups in the total cohort before and after propensity score matching. In the total cohort before matching, significant differences in LVEF and measures of structural heart disease are demonstrated (blue triangles). In the matched cohorts (red squares), these variable differences appear well matched. In our propensity match analysis, there were statistically significant differences in early survival, i.e. during the first 5 years (Figure 3, $\mathrm{p}=0.05$ ) between patients on amiodarone compared to patients on no AADs and non-amiodarone AAD groups, however these differences were not significant over longer term follow up ( $\mathrm{p}=0.08$ ). Survival estimates at 1,5 and 10 years for the 130 matched patients on non-amiodarone AADs were $97 \%, 78 \%$, and $45 \%$; and on amiodarone $86 \%, 63 \%$, and $41 \%$, respectively.

Propensity matching was also performed in amiodarone vs non-amiodarone AAD groups without LVH. There were 114 well matched pairs found. Kaplan-Meier survival curves in the propensity score matched 114 amiodarone treated and 114 non-amiodarone AAD treated patients with no LVH showed no significant survival differences between amiodarone and non-amiodarone antiarrhythmic drugs. Survival estimates at 1, 5 and 10 years for the 114 
matched patients on non-amiodarone AADs were $96 \%, 80 \%$, and $58 \%$; and on amiodarone $91 \%, 70 \%$, and $46 \%$, respectively.

\section{DISCUSSION}

In this study of the use of AADs among pts with or without LVH and AF, our principle findings were: 1) Patients on non-amiodarone AADs, with or without LVH had better survival when compared to patients on amiodarone in unadjusted analyses; 2) After adjusting for differences in these groups in propensity matched analyses, early survival differences were noted, worse for patients on amiodarone when compared to patients who were not on AADs or on non-amiodarone AADs; however, these differences were not noted in with longer term (>8 years) follow up.

Therapeutic options for AF have evolved with the development of pulmonary vein ablation over the past decade. However, a meta-analysis showed that single pulmonary vein isolation procedures achieve successful rhythm control off of AADs in only 57\% of patients, and multiple pulmonary vein ablations were successful in $71 \%$ of patients off of AADs; therefore adjunctive use of AADs remain an important option for many patients with symptomatic AF in the post ablation period, as well as remaining a primary therapeutic option for many other patients. ${ }^{10}$ The choice of AADs is often limited and based on co-morbid conditions.

One of these comorbid conditions is the presence of LVH. LVH has long been associated with increased risk of sudden cardiac death. ${ }^{11-13}$ It has been proposed that patients with hypertension and LVH are at increased risk of torsades de pointes because of a predisposition to the development of early afterdepolarizations. ${ }^{14-16}$ Two animal studies have suggested that hypertrophied hearts in dogs have increased risks of proarrhythmia with dofetilide and BAY K 8644, a calcium channel activator. These drugs increased transmural dispersion of ventricular repolarization and induced early phase 2 afterdepolarizations, resulting in easily inducible ventricular tachyarrhythmias. ${ }^{14-17}$ Prior animal models with cardiac hypertrophy have also demonstrated that LVH has pronounced effects on dispersion of refractoriness and repolarization, increasing vulnerability to fibrillation. ${ }^{18}$

Although AF guidelines have recommended amiodarone as a drug of choice in patients with $\mathrm{AF}$ and substantial LVH, ${ }^{6-8}$ This recommendation poses a significant challenge in many patients with $\mathrm{LVH}$ who might be at risk for long term toxic effects of amiodarone. ${ }^{19-22} \mathrm{~A}$ rationale for amiodarone may be in its perceived lower risk of proarrhythmia. Although amiodarone may prolong QT interval, its propensity for torsades de pointes is exceedingly low, possibly due to multiple ion channel blockade that may reduce early afterdepolarizations and/or reduced transmural dispersion of repolarization. ${ }^{23,}{ }^{24}$ Similarly dronedarone, approved by the Federal Drug Administration for use in the United States in 2009, is also a multi-channel blocker and was added to the algorithm for patients with $\mathrm{LVH}$ and AF by the ESC 2010 AF guidelines. ${ }^{825}$

However, to our knowledge, there is limited clinical data in humans to guide AAD recommendations in patients with $\mathrm{AF}$ and $\mathrm{LVH}$, as there has been no clinical study of safety or survival outcomes comparing the use of AADs in the presence of $\mathrm{LVH}$ and $\mathrm{AF}$, including 
prior pivotal AF trials such as AFFIRM and the Canadian Trial of Atrial Fibrillation. ${ }^{26}$ The AFFIRM AAD substudy noted more deaths in patients who were randomly assigned to Class 1 AADs compared to amiodarone, although most of the deaths occurred after change of the initial AAD assignment, amiodarone was often the replacing drug, and LVH was not addressed. ${ }^{27}$ In another sub-analysis of AFFIRM, amiodarone, sotalol or class 1C AADs were compared to propensity-score matched rate control patients. The composite outcome of mortality and first cardiovascular hospitalization was significantly higher in the amiodarone and sotalol groups, but not significantly different in the Class $1 \mathrm{C}$ group. In this study the AAD groups were not compared against one another nor was any analysis done specific to LVH. ${ }^{28}$ Indeed, the Canadian Cardiovascular Society Atrial Fibrillation Guidelines 2010 recognized the weakness of the scientific data supporting AAD recommendations in $\mathrm{LVH}$ and advised that the choice of AADs in patients with LVH be individualized. ${ }^{25,} 29$

The current analysis studied overall mortality among patients with persistent AF and LVH comparing various AADs to determine whether differences in survival between amiodarone and non-amiodarone AADs could be detected to substantiate AF guideline recommendations. The primary findings of a trend toward lower survival on amiodarone were noted. The lower survival on amiodarone in unadjusted Kaplan-Meier and adjusted Cox proportional hazards analyses cannot be viewed as causal, as the association was at least in part due to incomplete adjustment for the higher incidence of CAD and other structural heart disease in amiodarone-treated patients in this non-randomized study. However, it should be noted that CAD itself was not a predictor of mortality in our Cox proportional hazard model. The significant baseline differences in the amiodarone treated group are expected, since amiodarone is commonly reserved for patients with structural heart disease or more refractory cases of AF. To further adjust for baseline differences and use of amiodarone, a more robust matched group analysis was performed, adjusting for propensity scores. In this analysis, there was a trend toward lower survival in the amiodarone group in the first few years of drug exposure. Although there was a trend toward a higher early mortality on amiodarone, long-term survival was not significantly different, but neither was long term survival better on amiodarone to support amiodarone as a superior AAD choice.

Conversely, Class 1C drugs were associated with better survival compared to amiodarone in patients with LVH (adjusted $\mathrm{p}=0.032$ ). This result also reflects the common use of Class 1C drugs in patients without significant structural heart disease compared to amiodarone, which is more commonly used in the presence of structural heart disease.

It should be emphasized that the current analysis was based on AAD use at the time of initial cardioversion and at a time when dofetilide was not used widely in this population in part due to adherence to guidelines recommendations and also as it was newly approved in 1999 in the United States during the study period. Thus, the principal conclusion that can be drawn from this long-term analysis is that mortality outcomes are better in patients with persistent $\mathrm{AF}$ who are deemed appropriate for non-amiodarone AADs despite the presence of LVH and are cardioverted, compared to matched patients who are deemed appropriate for amiodarone therapy and cardioverted. Overall, this study certainly did not show a survival benefit in a cohort of patients with persistent $\mathrm{AF}$ with $\mathrm{LVH}$ on amiodarone. 


\section{STUDY LIMITATIONS}

This was a single center observational cohort analysis in a tertiary referral care center which partly explains a higher incidence of CAD and other structural heart disease compared to other multicenter AF trial such as AFFIRM. Therefore, results may not be representative to other medical centers and may limit applicability to other populations. Secondly, the nature of this retrospective observational analysis with a heterogeneous population incurs confounding variables despite our best efforts to identify baseline differences, which also creates a significant limitation to application in the AF population with $\mathrm{LVH}$ at large. We attempted to analyze comparable groups using propensity score matching; however, propensity scores may not have adjusted for all inherent clinical variables that determined AAD usage. Further, we were only able to analyze by the antiarrhythmic drug taken at the time of cardioversion. AAD use is often dynamic over the lifetime of a patient with AF, but monitoring each drug use over time on each patient was not feasible in this study. We view our analysis as analogous to an intention to treat design in which patients with protocol deviations or lost to follow up remain included in the analysis. Finally, the use of dronedarone could not be addressed in this study, as dronedarone was unavailable during the study period.

\section{CONCLUSIONS}

Results of this study highlight the dilemma we have in considering amiodarone as the main $\mathrm{AAD}$ of choice in patients with persistent $\mathrm{AF}$ and $\mathrm{LVH}$. The findings of this retrospective observational study merits further prospective evaluation in a randomized fashion.

Nevertheless, our findings are discordant with current management guidelines recommending amiodarone and excluding $1 \mathrm{C}$ or non-amiodarone class III AADs for this cohort. Unless further randomized trials become available, clinical decisions on AAD use in patients with persistent AF and LVH should be individualized with prudent considerations for use of amiodarone vs. non-amiodarone AADs.

\section{ACKNOWLEDGEMENTS}

The authors gratefully acknowledge the graphics assistance of Suzanne Turner from the Cleveland Clinic Cardiovascular Medicine office of Graphics and Design.

Dr Mina Chung reports NIH grant support (R01 HL090620 and R01 HL111314).

\section{REFERENCES}

1. Kannel WB, Wolf PA, Benjamin EJ, Levy D. Prevalence, incidence, prognosis, and predisposing conditions for atrial fibrillation: population-based estimates. Am J Cardiol. 1998; 82:2N-9N.

2. Healey JS, Connolly SJ. Atrial fibrillation: hypertension as a causative agent, risk factor for complications, and potential therapeutic target. Am J Cardiol. 2003; 91:9G-14G.

3. Lau DH, Mackenzie L, Kelly DJ, Psaltis PJ, Worthington M, Rajendram A, Kelly DR, et al. Shortterm hypertension is associated with the development of atrial fibrillation substrate: a study in an ovine hypertensive model. Heart Rhythm. 2010; 7:396-404. [PubMed: 20185115]

4. Benjamin EJ, Levy D, Vaziri SM, D'Agostino RB, Belanger AJ, Wolf PA. Independent risk factors for atrial fibrillation in a population-based cohort. The Framingham Heart Study. JAMA. 1994; 271:840-844. [PubMed: 8114238] 
5. Camm AJ. Safety considerations in the pharmacological management of atrial fibrillation. Int J Cardiol. 2008; 127:299-306. [PubMed: 18191470]

6. Fuster V, Ryden LE, Cannom DS, Crijns HJ, Curtis AB, Ellenbogen KA, Halperin JL, et al. ACC/AHA/ESC 2006 Guidelines for the Management of Patients with Atrial Fibrillation: a report of the American College of Cardiology/American Heart Association Task Force on Practice Guidelines and the European Society of Cardiology Committee for Practice Guidelines (Writing Committee to Revise the 2001 Guidelines for the Management of Patients With Atrial Fibrillation): developed in collaboration with the European Heart Rhythm Association and the Heart Rhythm Society. Circulation. 2006; 114:e257-354. [PubMed: 16908781]

7. Wann LS, Curtis AB, January CT, Ellenbogen KA, Lowe JE, Estes NA 3rd, Page RL, et al. ACCF/AHA/HRS focused update on the management of patients with atrial fibrillation (Updating the 2006 Guideline): a report of the American College of Cardiology Foundation/American Heart Association Task Force on Practice Guidelines. J Am Coll Cardiol 2011. 2011; 57:223-242.

8. European Heart Rhythm Association, European Association for Cardio-Thoracic Surgery. Camm AJ, Kirchhof P, Lip GY, Schotten U, Savelieva I, Ernst S, Van Gleder I, et al. Guidelines for the management of atrial fibrillation: the Task Force for the Management of Atrial Fibrillation of the European Society of Cardiology (ESC). Eur Heart J. 2010; 31:2369-2429. [PubMed: 20802247]

9. Fuster V, Ryden LE, Asinger RW, Cannom DS, Crijns HJ, Frye RL, Halperin JL, et al. ACC/AHA/ESC Guidelines for the Management of Patients With Atrial Fibrillation: Executive Summary A Report of the American College of Cardiology/American Heart Association Task Force on Practice Guidelines and the European Society of Cardiology Committee for Practice Guidelines and Policy Conferences (Committee to Develop Guidelines for the Management of Patients With Atrial Fibrillation) Developed in Collaboration With the North American Society of Pacing and Electrophysiology. Circulation. 2001; 104:2118-2150. [PubMed: 11673357]

10. Calkins H, Reynolds MR, Spector P, Sondhi M, Xu Y, Martin A, Williams CJ, et al. Treatment of atrial fibrillation with antiarrhythmic drugs or radiofrequency ablation: two systematic literature reviews and meta-analyses. Circ Arrhythm Electrophysiol. 2009; 2:349-361. [PubMed: 19808490]

11. Kannel WB, Gordon T, Offutt D. Left ventricular hypertrophy by electrocardiogram. Prevalence, incidence, and mortality in the Framingham study. Ann Intern Med. 1969; 71:89-105. [PubMed: 4239887]

12. Zipes DP, Wellens HJ. Sudden cardiac death. Circulation. 1998; 98:2334-2351. [PubMed: 9826323]

13. Levy D, Garrison RJ, Savage DD, Kannel WB, Castelli WP. Prognostic implications of echocardiographically determined left ventricular mass in the Framingham Heart Study. N Engl J Med. 1990; 322:1561-1566. [PubMed: 2139921]

14. Ben-David J, Zipes DP, Ayers GM, Pride HP. Canine left ventricular hypertrophy predisposes to ventricular tachycardia induction by phase 2 early afterdepolarizations after administration of BAY K 8644. J Am Coll Cardiol. 1992; 20:1576-1584. [PubMed: 1280660]

15. Sipido KR, Volders PG, de Groot SH, Verdonck F, Van de Werf F, Wellens HJ, Vos MA. Enhanced $\mathrm{Ca}(2+)$ release and $\mathrm{Na} / \mathrm{Ca}$ exchange activity in hypertrophied canine ventricular myocytes: potential link between contractile adaptation and arrhythmogenesis. Circulation. 2000; 102:2137-2144. [PubMed: 11044433]

16. Wolk R. Arrhythmogenic mechanisms in left ventricular hypertrophy. Europace. 2000; 2:216-223. [PubMed: 11227591]

17. Kozhevnikov DO, Yamamoto K, Robotis D, Restivo M, El-Sherif N. Electrophysiological mechanism of enhanced susceptibility of hypertrophied heart to acquired torsade de pointes arrhythmias: tridimensional mapping of activation and recovery patterns. Circulation. 2002; 105:1128-1134. [PubMed: 11877367]

18. Kowey PR, Friechling TD, Sewter J, Wu Y, Sokil A, Paul J, Nocella J. Electrophysiological effects of left ventricular hypertrophy. Effect of calcium and potassium channel blockade. Circulation. 1991; 83:2067-2075. [PubMed: 1645624]

19. Vorperian VR, Havighurst TC, Miller S, January CT. Adverse effects of low dose amiodarone: a meta- analysis. J Am Coll Cardiol. 1997; 30:791-798. [PubMed: 9283542]

20. Harris L, McKenna WJ, Rowland E, Holt DW, Storey GC, Krikler DM. Side effects of long-term amiodarone therapy. Circulation. 1983; 67:45-51. [PubMed: 6291807] 
21. Batcher EL, Tang XC, Singh BN, Singh SN, Reda DJ, Hershman JM. SAFE-T Investigators. Thyroid function abnormalities during amiodarone therapy for persistent atrial fibrillation. Am $\mathrm{J}$ Med. 2007; 120:880-885. [PubMed: 17904459]

22. Dusman RE, Stanton MS, Miles WM, Klein LS, Zipes DP, Fineberg NS, Heger JJ. Clinical features of amiodarone-induced pulmonary toxicity. Circulation. 1990; 82:51-59. [PubMed: 2364524]

23. Kannankeril PJ, Roden DM. Drug-induced long QT and torsade de pointes: recent advances. Curr Opin Cardiol. 2007; 22:39-43. [PubMed: 17143043]

24. van Opstal JM, Schoenmakers M, Verduyn SC, de Groot SH, Leunissen JD, van Der Hulst FF, Molenschot MM, et al. Chronic amiodarone evokes no torsade de pointes arrhythmias despite QT lengthening in an animal model of acquired long-QT syndrome. Circulation. 2001; 104:27222727. [PubMed: 11723026]

25. Gillis AM, Verma A, Talajic M, Nattel S, Dorian P. CCS Atrial Fibrillation Guidelines Committee. Canadian Cardiovascular Society atrial fibrillation guidelines 2010: rate and rhythm management. Can J Cardiol 2011. 27:47-59.

26. Roy D, Talajic M, Dorian P, Connolly S, Eisenberg MJ, Green M, Kus T, et al. Amiodarone to prevent recurrence of atrial fibrillation. Canadian Trial of Atrial Fibrillation Investigators. N Engl J Med. 2000; 342:913-920. [PubMed: 10738049]

27. AFFIRM First Antiarrhythmic Drug Substudy Investigators. Maintenance of sinus rhythm in patients with atrial fibrillation: an AFFIRM substudy of the first antiarrhythmic drug. J Am Coll Cardiol. 2003; 42:20-29. [PubMed: 12849654]

28. Saksena S, Slee A, Waldo AL, Freemantle N, Reynolds M, Rosenberg Y, Rathod S, et al. Cardiovascular outcomes in the AFFIRM Trial (Atrial Fibrillation Follow-Up Investigation of Rhythm Management). An assessment of individual antiarrhythmic drug therapies compared with rate control with propensity score-matched analyses. J Am Coll Cardiol. 2011; 58:1975-1985. [PubMed: 22032709]

29. Skanes AC, Healey JS, Cairns JA, Dorian P, Gillis AM, McMurtry MS, Mitchell LB, et al. Focused 2012 update of the Canadian Cardiovascular Society atrial fibrillation guidelines: recommendations for stroke prevention and rate/rhythm control. Can J Cardiol 2012. 28:125-136. 
A.

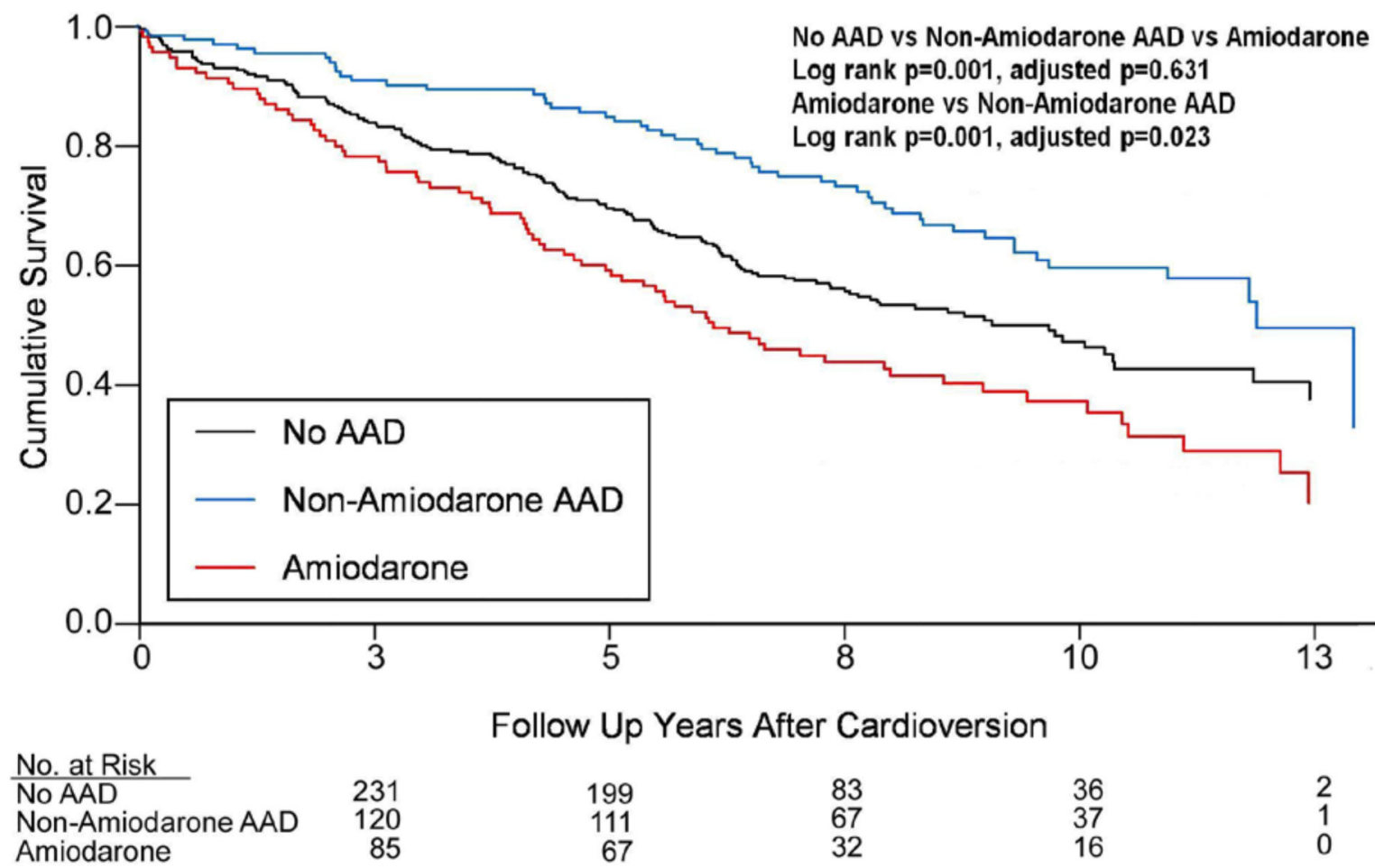


B

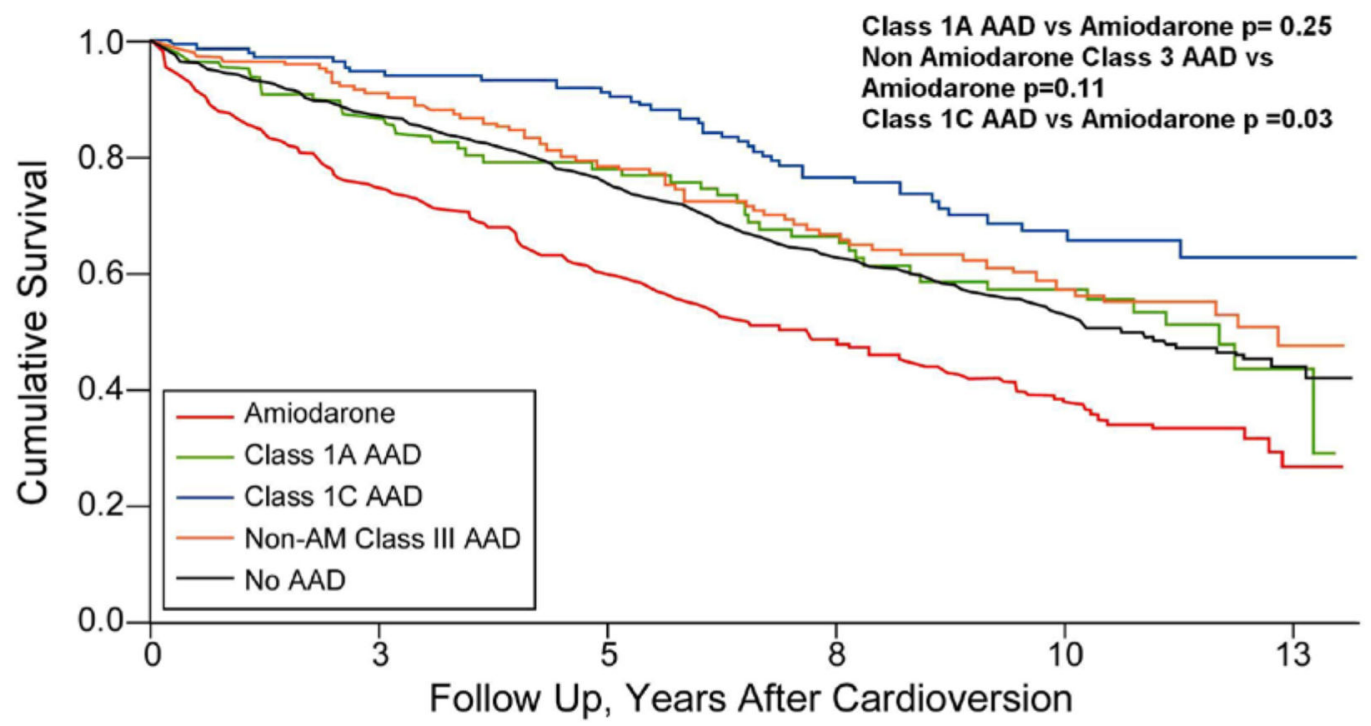

No. at Risk

Amiodarone 111

Class 1A AAD 31

Class 1C AAD 47

Non-AM Class III AAD 50

No AAD

288

80
25
45
43
230

63
24
44
38
200

45
21
36
31
156

42
21
32
27
147

Figure 1.

Kaplan Meier survival analysis stratified by antiarrhythmic drug (AAD) use in patients with LVH ( $\geq 1.4 \mathrm{~cm}$ septal or posterior wall thickness). A. Analysis in patients stratified by no antiarrhythmic drug, amiodarone, and non-amiodarone antiarrhythmic drug use. B. Analysis in patients stratified by no AAD, class IA, class $1 \mathrm{C}$, amiodarone, and non-amiodarone class III (Non-AM Class III) AAD use. 


\section{Covariate Balance}

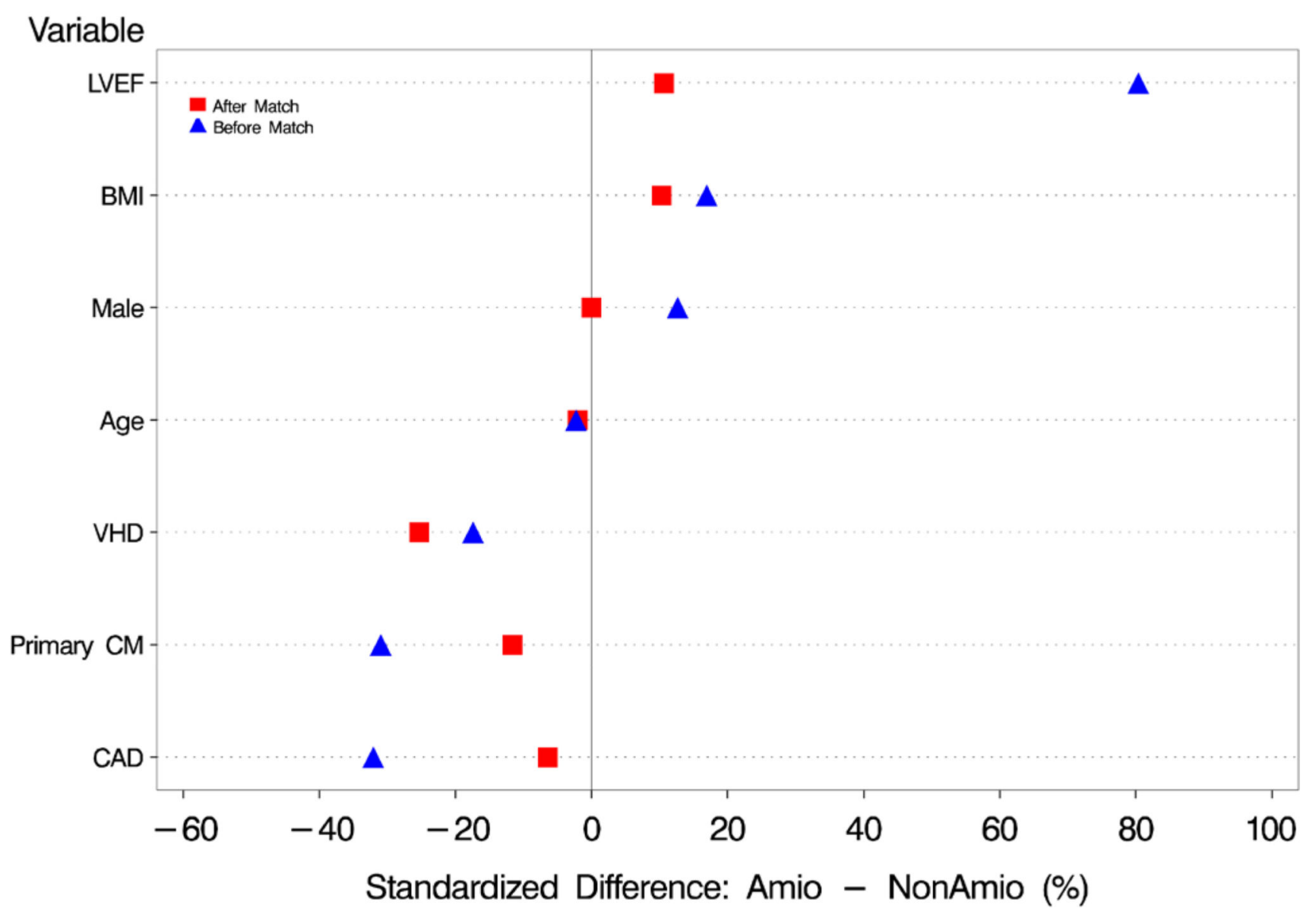

Figure 2.

Covariance balance plot showing standardized differences between the amiodarone and nonamiodarone antiarrhythmic drug groups before and after propensity score matching. 


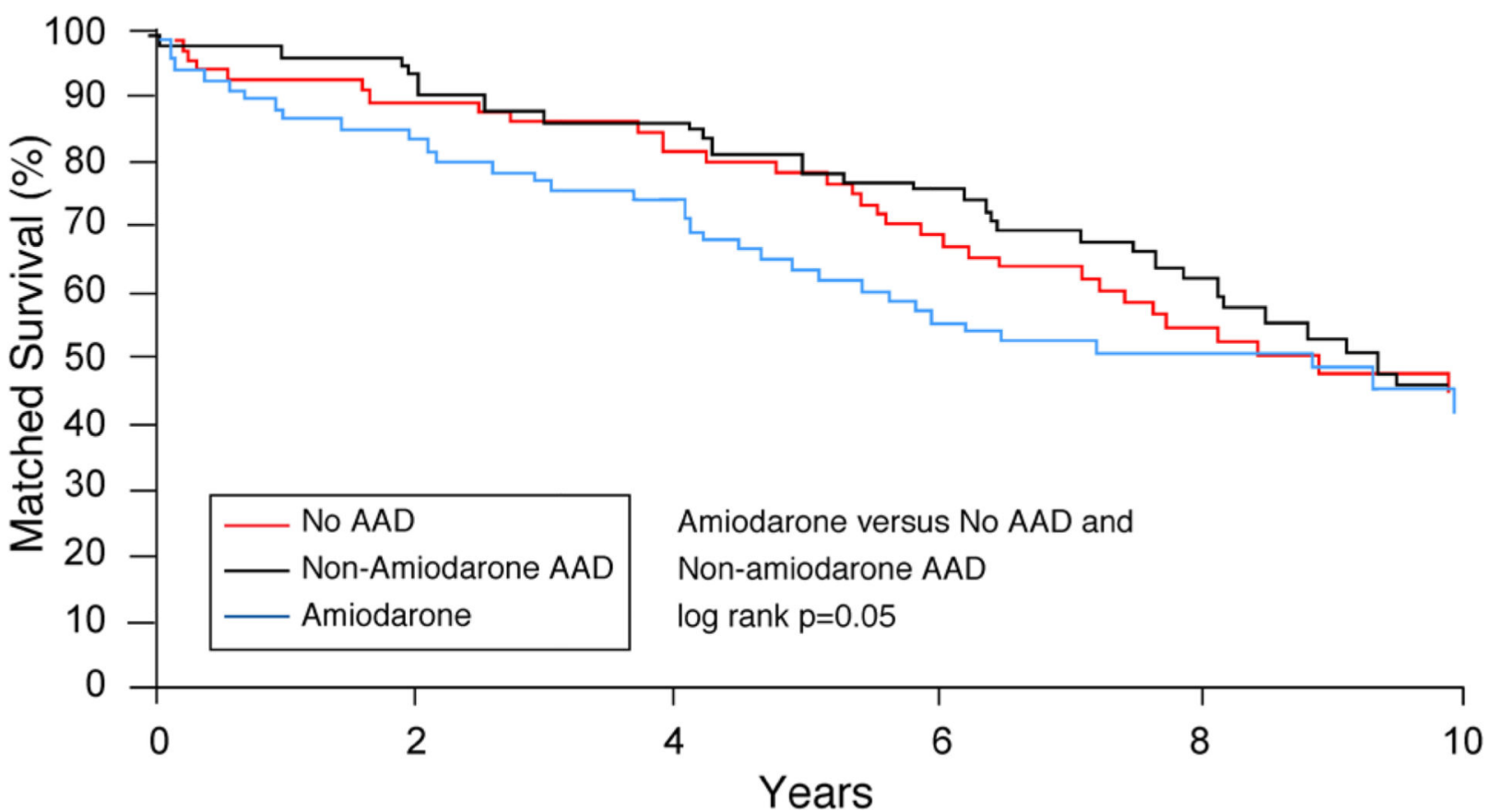

Figure 3.

Kaplan Meier survival analysis stratified by antiarrhythmic drug use in: A. Propensity matched patients with LVH (septal or posterior wall thickness $\geq 1.4 \mathrm{~cm}$ ). 


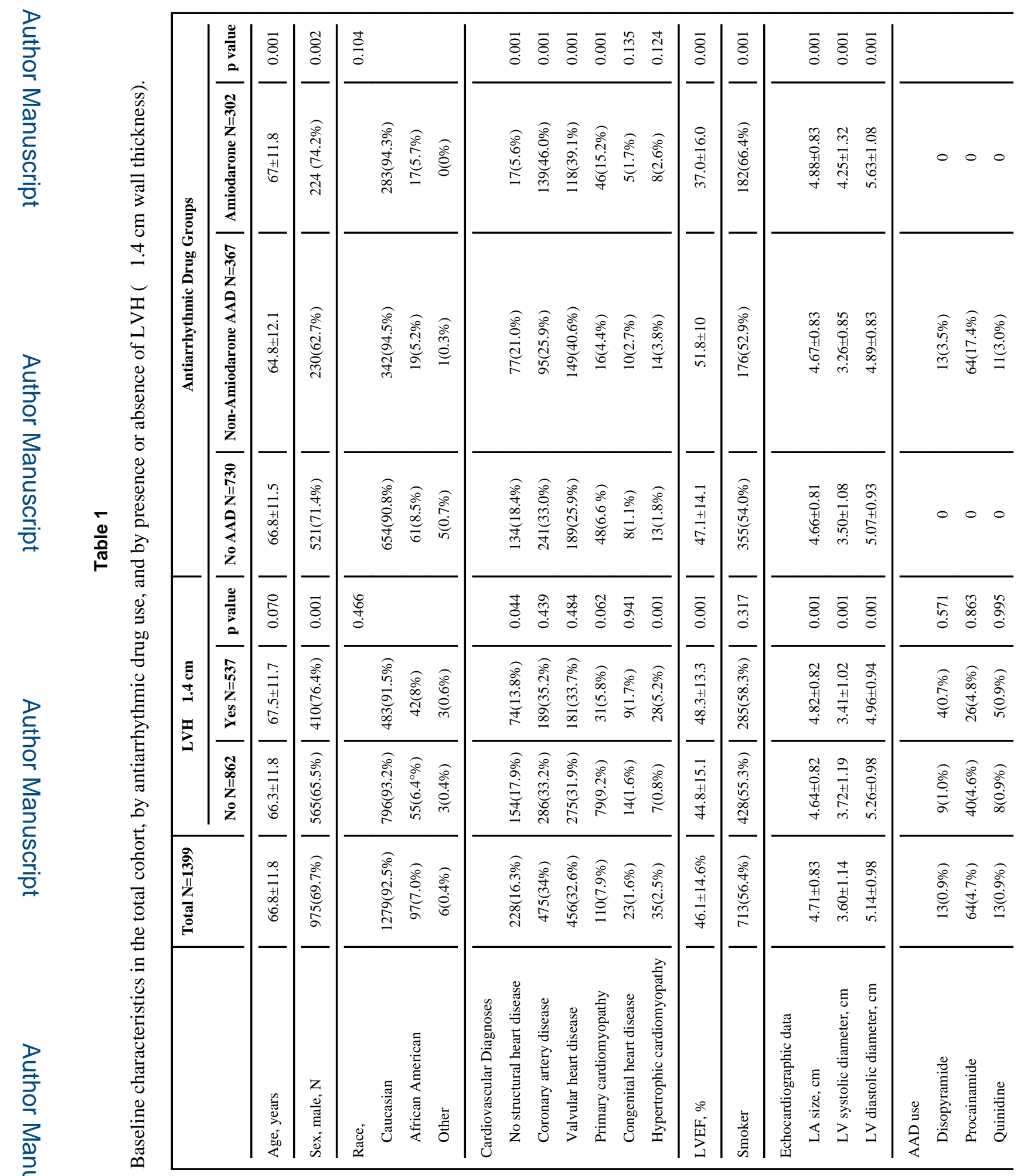

Pacing Clin Electrophysiol. Author manuscript; available in PMC 2015 October 01. 


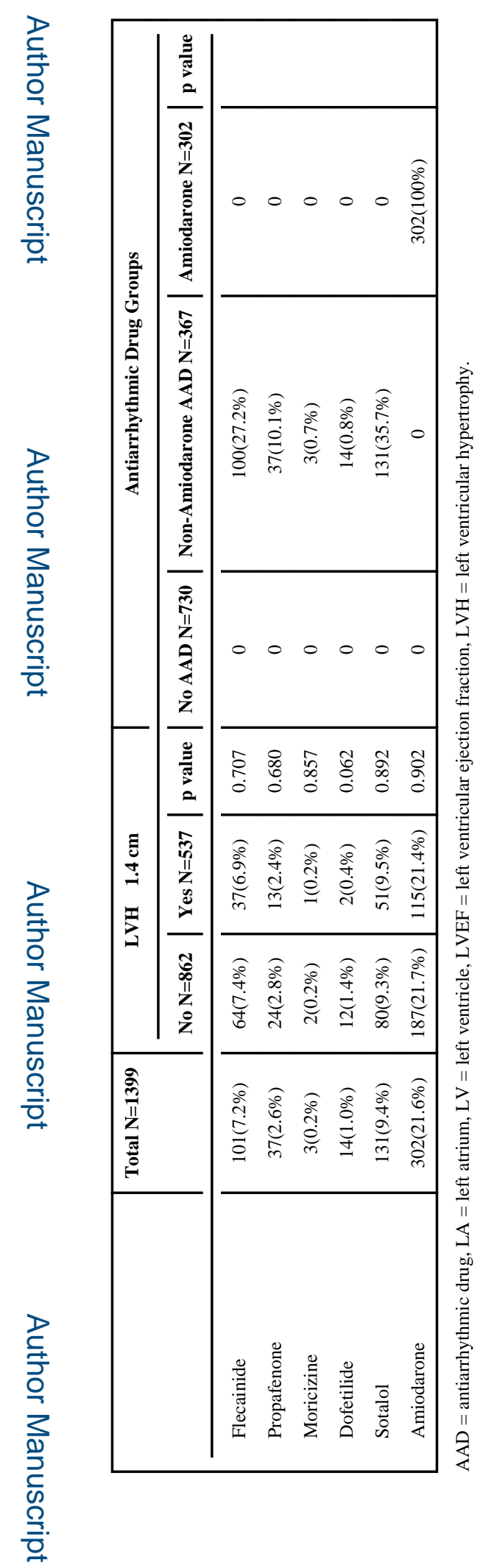

Pacing Clin Electrophysiol. Author manuscript; available in PMC 2015 October 01. 


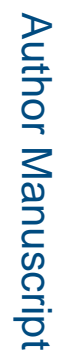

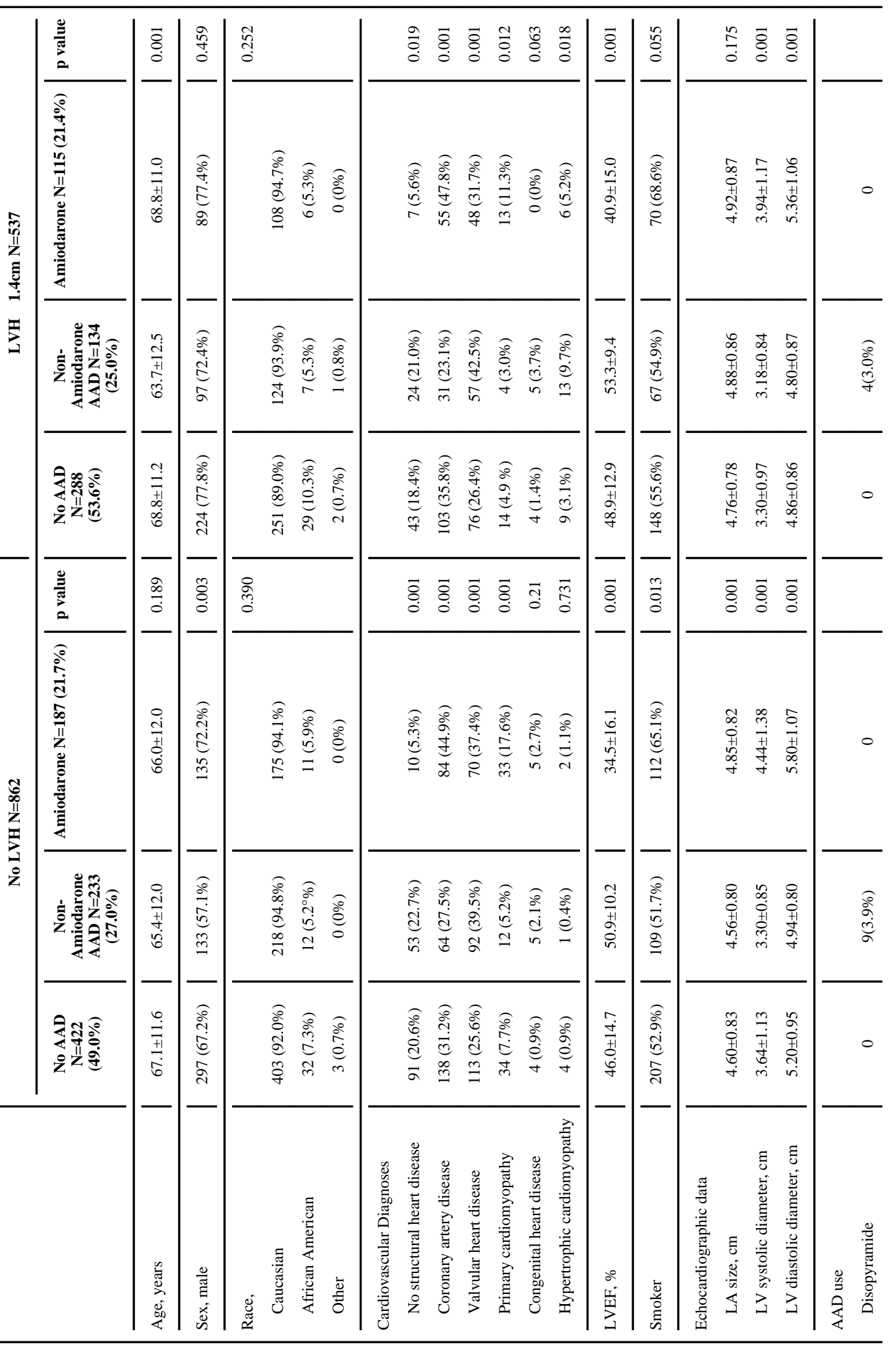

Pacing Clin Electrophysiol. Author manuscript; available in PMC 2015 October 01. 


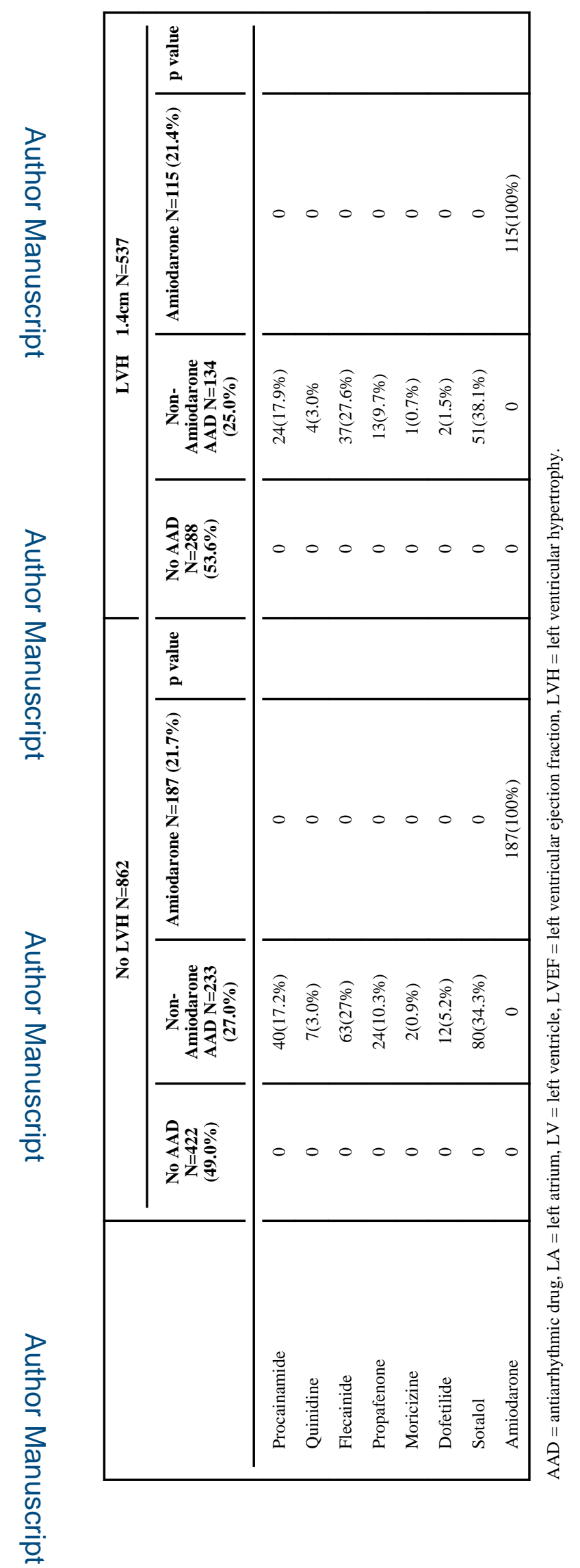

Pacing Clin Electrophysiol. Author manuscript; available in PMC 2015 October 01. 
Table 3

Predictors of mortality in patients with LVH on AADs by Cox proportional hazards modeling.

\begin{tabular}{|c|c|c|c|}
\hline Variable & Hazard ratio & 95\% Confidence interval & p value \\
\hline Age, yr & 1.051 & $1.030,1.072$ & 0.001 \\
\hline LVEF, \% & 0.982 & $0.968,0.996$ & 0.010 \\
\hline CAD & 0.973 & $0.652,1.451$ & 0.893 \\
\hline Smoker & 1.647 & $1.097,2.494$ & 0.016 \\
\hline Amiodarone & 1.655 & $1.072,2.555$ & 0.023 \\
\hline
\end{tabular}

$\mathrm{N}=249 . \mathrm{LVEF}=$ left ventricular ejection fraction; $\mathrm{CAD}=$ coronary artery disease. 
Table 4

Characteristics of patients in the propensity score matched LVH no antiarrhythmic drug group, amiodarone and non-amiodarone AAD groups.

\begin{tabular}{|l|c|c|c|c|}
\hline & No AAD N=65 (\%) & Non-Amiodarone N=65 (\%) & Amiodarone N=65 (\%) & P-value \\
\hline Sex, male & $17(26)$ & $16(25)$ & $16(25)$ & .97 \\
\hline Race, Caucasian & $61(95)$ & $63(97)$ & $63(97)$ & .99 \\
\hline Age, years & $68.9 \pm 12.6$ & $67.7 \pm 9.96$ & $67.7 \pm 9.96$ & 0.49 \\
\hline Height, cm & $174 \pm 11.6$ & $175 \pm 11.8$ & $175 \pm 11.8$ & 0.87 \\
\hline Weight, kg & $86.1 \pm 20.6$ & $89.8 \pm 21.4$ & $89.8 \pm 21.4$ & 0.89 \\
\hline Body mass index & $28.6 \pm 5.85$ & $29.3 \pm 6.3$ & $29.3 \pm 6.3$ & 0.86 \\
\hline Coronary Artery Diseas & $25(38)$ & $23(35)$ & $25(38)$ & .92 \\
\hline Valvular heart disease & $31(48)$ & $22(34)$ & $30(46)$ & .22 \\
\hline Primary Cardiomyopathy & $8(12)$ & $4(6.2)$ & $6(9.2)$ & .48 \\
\hline Hypertrophic cardiomyopathy & $3(4.6)$ & $5(7.7)$ & $3(4.6)$ & .68 \\
\hline History of alcohol use & $8(21)$ & $15(23)$ & $15(23)$ & .94 \\
\hline Smoker & $18(46)$ & $39(61)$ & $39(60)$ & .94 \\
\hline LVEF, \% & $49.8 \pm 13.4$ & $50.1 \pm 11$ & $50.1 \pm 11$ & 0.74 \\
\hline LV posterior wall thickness, cm & $1.42 \pm 0.35$ & $1.39 \pm 0.24$ & $1.39 \pm 0.24$ & 0.97 \\
\hline LV septal thickness, cm & $1.61 \pm 0.36$ & $1.65 \pm 0.72$ & $1.65 \pm 0.72$ & 0.59 \\
\hline
\end{tabular}

$\mathrm{LVEF}=$ left ventricular ejection fraction, $\mathrm{LV}=$ left ventricle 